\title{
Analysis of a method for monitoring multi-nozzle-arrays
}

\author{
Florian Schulz ${ }^{* 1}$, Franziska Reincke ${ }^{1}$, Matthias Mrochen ${ }^{2}$, Frank Beyrau ${ }^{1}$ \\ ${ }^{1}$ Lehrstuhl für Technische Thermodynamik, Universität Magdeburg, Germany \\ ${ }^{2}$ Fimro GmbH, Langenstein, Germany \\ *Corresponding author email : florian.schulz@ovgu.de
}

\begin{abstract}
In casting technology spraying tools are used for cooling and cleaning purposes. Its heavy use is often accompanied by contamination and misalignment of the nozzles, which leads to the production of rejects and to production downtime. Therefore, control systems are necessary to check the function of the nozzles and in case of a problem to find the defect. From this starting point, we develop a new robust measuring technique.

This measurement technic is based on the optical visualisation of the spray impingement on a frosted pane. A camera detects the resulting characteristic wetting pattern. From the camera recordings, conclusions can be drawn about the alignment and the mass flow of individual nozzles. To test the capabilities of the measurement method, we used a spray tool with 9 nozzles and equipped with different nozzles as shown in Figure 1. For this we have measure the position of spray impact and the size of the wetted area of the individual spray plumes on the frosted pane surface. As a result, we are able to show that the measurement results are reproducible and the measurement method achieves high sensitivity.
\end{abstract}

\section{Keywords}

spray monitoring, wetting pattern, multi nozzle array, function control

\section{Introduction}

In foundry technology, large nozzle arrays are used, for example, for cooling, cleaning or release agent wetting. During its usage under harsh conditions, the nozzles may show contamination or changes in geometric alignment. In turn, this causes changes in the positioning of the spray or changes of the amount and distribution of the liquid on the target surface. Such changes are usually only noticed when the production of rejects occurs, with the consequence of production downtimes. To avoid such failures, there is a need for measurement systems to monitor the process sprays. Process control enables improvement of product quality and reduction of rejects [1]. As part of process control, the nozzle performance should be regularly monitored. This can be done by characterizing the spray [2].
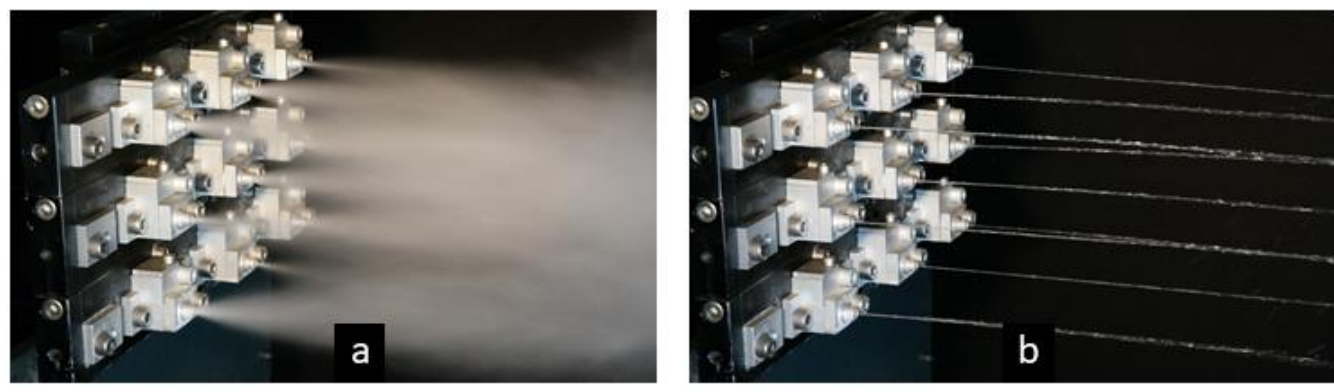

Figure 1. a) Complex Spray of the multi-nozzle spraying tool applied in this study b) operating mode of straight water injection. 
In particular, optical measurement techniques are suitable for characterizing complex processes because they can provide a wide range of information, have high temporal and spatial resolution, and provide a rapid overview of phenomena that occur [3].

Common methods are designed to determine droplet sizes and velocities, like PDA or PIVtechnique. The use of these techniques in manufacturing facilities is associated with various disadvantages, including the high cost of lasers, the sensitivity of optics to contamination, or the necessary safety requirements for operation.

Spray patternators are another way to characterize a spray by its mass distribution. However, For the considered application with large nozzle arrays it is very time consuming to achieve the necessary high local resolution and high accuracy in local water mass determination [4]. For the characterization of the entire spray of large multi-nozzle spraying tools, there is currently no measurement method that allows a fast and efficient recording of the entire spray. For this reason, we have investigated a method that makes this possible. The method is based on wetting the surface of a roughened transparent plate [5], where the detectable light scattering from the rough surface changes in the areas of liquid wetting. The wetting pattern created during injection can be detected with a conventional camera. It can be assumed that the wetting pattern of a nozzle is characteristic for the generated spray, like a fingerprint. Small changes in the spray would also result in a change in the wetting pattern. For many applications, it is critical that the spray is the desired, adjusted state. Changes in the desired state often cause problems, for example, when a surface needs to be uniformly wetted with release agent. The described measurement technique now allows a master recording of the target state of the spray to be taken. This can be compared at any later time with a control image of the current actual state. Deviations between the recordings indicate changes in the spray and thus the need to control and correct the spraying tool. Ideally, the measurement technique allows direct conclusions to be drawn about the cause of the spray changes. Narrowing down the causes of spray changes is a major benefit of the method described. In the following, the wetting pattern method will be examined in more detail, focusing on the evaluation of the positions of individual wetting areas and the size of the wetting areas using different operating modes of the spraying tool.

\section{Material and Methods}

The basis of the measuring system is a camera that records the surface of a frosted pane. As shown in Figure 2 (left), the spraying tool is located on the opposite side of the pane. The spraying tool used for the study has an array of $3 \times 3$ nozzles. This makes it comparable to nozzle arrays used in foundry industry. The nozzles used are externally mixing air-water nozzles. In this case, the atomization of the water jet is achieved by the parallel injection of air jets. Figure 2 (right) shows the channel arrangement, with the air bearing holes in the circumference around the water bearing hole in the center.

In practice, the nozzle arrays can be equipped with different types of nozzles. To take this into account in the present study, two different nozzles were used. Six nozzles with large hole diameter and three nozzles with small hole diameter. The positioning within the nozzle array is shown in Figure 2 by dots of different sizes.

Preliminary investigations have shown that a small distance between nozzle array and frosted pane is advantageous. For the present investigation the distance between the two was set to $11 \mathrm{~cm}$. In this measurement campaign, the air pressure is set to 5 bar and the water pressure to 4 bar. 

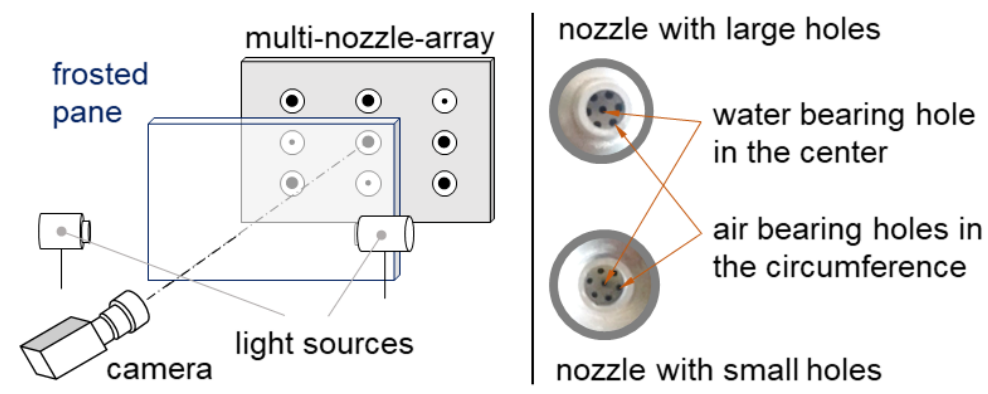

Figure 2. Wetting pattern method setup (left) and images of two different nozzle (right).

The spraying tool allows the compressed air and the water to be controlled separately. This makes it possible to examine the function of the water-carrying and the air-carrying system separately. If only water is injected, this is referred to in the following as "straight water injection". If air is also injected and the water is atomized, this is referred to in the following as "spray injection". The resulting jets of these two injection types are shown in Figure 1.

One advantage of the measurement technique is that a commercially available camera can be used. In this study, a Basler Ace acA1300-200 $\mu \mathrm{m}$ was applied in combination with a wideangle lens. With a camera resolution of $1280 \times 1024$, this combination results in an image resolution of $0.2 \mathrm{~mm} /$ pixel. LEDs with appropriate electronics to eliminate current fluctuations were used as light source. At the set exposure time of $1 \mathrm{~ms}$, wetting patterns result as shown in Figure 3. Detailed information about the image processing can be found in [6].
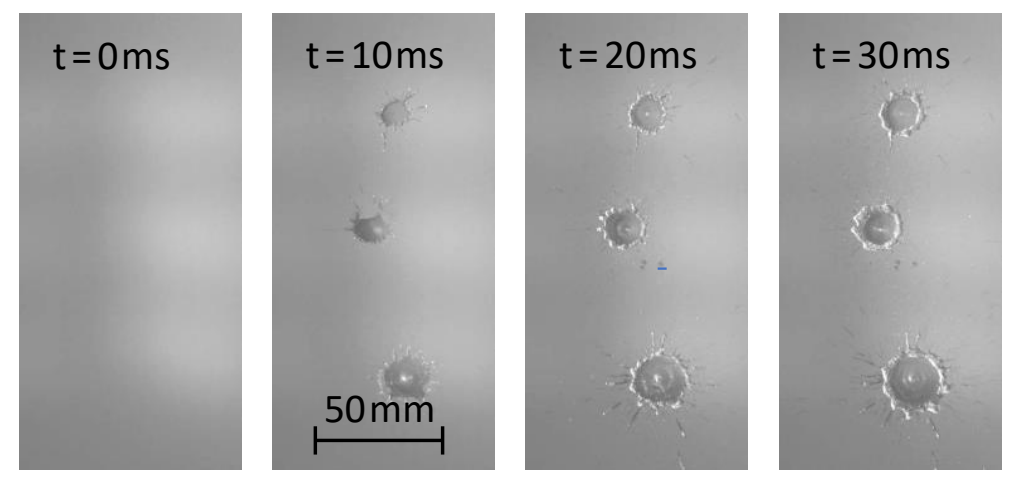

Figure 3. Time series of wetting patterns of three out of nine nozzles.

\section{Results and Discussion}

From the scattered light images, two main parameters can be determined from the individual wetted areas, the position of the centers of the wetted areas and the size of the wetted areas. The accuracy of the determination of the positions is much higher than the local image resolution, which was proven in a previous study. A similar effect occurs with the size of the areas, above a certain camera resolution a further refinement of the resolution hardly leads to a higher accuracy of the calculated area values. For the application of the proposed measurement technique, it is important to know how much the parameters vary from injection to injection; that is, whether the measurements are reproducible. If so, the method can be used to detect undesirable changes in the spraying tool. For this reason, the reproducibility of the measurement results of the center positions and the areas will be examined in more detail below. 


\section{Monitoring of the Nozzle Alignment}

The process until all nine jets are wetting the frosted pane takes up to $40 \mathrm{~ms}$. The image evaluation then starts after all jets have wetted the surface. First, the measurements are made using the straight water injection and afterwards using the spray injection. The experiments were repeated 10 times each.

The positions of the center points and the associated standard deviations occurring during repetition were calculated from the images and are displayed in Table 1. It can be seen that the largest variations occurred at the nozzle in the 1 column and 3 row. The standard deviation in vertical direction was $0.35 \mathrm{~mm}$ and in horizontal direction $0.33 \mathrm{~mm}$. These low values for the standard deviation are a proof for the reproducibility of the wetting pattern and in particular of the measurements of the center points in straight water injection.

For practical applications where a master recording is compared with a single test recording, a deviation of a center position of more than $2 \sigma$, i.e. $0.7 \mathrm{~mm}$, can be used to conclude that a change, such as misalignment or clogging, is present in the nozzle in a specific case. In our experience, this high sensitivity is much higher than required in practical applications. However, in any case, the sensitivity is higher than is made possible by visual adjustment by a technician.

Table 1 - Standard deviations of the positions of the centers of the wetted surfaces for straight water injection.

\begin{tabular}{cccll}
\hline column & row & nozzle holes & $\sigma_{\text {hor }}[\mathrm{mm}]$ & $\sigma_{\text {vert }}[\mathrm{mm}]$ \\
\hline \multirow{2}{*}{1} & 1 & large & 0.21 & 0.31 \\
& 2 & small & 0.17 & 0.25 \\
& 3 & large & $\mathbf{0 . 3 3}$ & $\mathbf{0 . 3 5}$ \\
\hline \multirow{2}{*}{2} & 1 & large & 0.22 & 0.22 \\
& 2 & large & 0.24 & 0.26 \\
& 3 & small & 0.31 & 0.23 \\
\hline \multirow{3}{*}{3} & 1 & small & 0.23 & 0.22 \\
& 2 & large & $\mathbf{0 . 1 2}$ & 0.20 \\
& 3 & large & 0.20 & $\mathbf{0 . 1 4}$ \\
\hline
\end{tabular}

When comparing the results of the straight water injection with further measurements using the spray injection it turns out that the midpoints of both working modes do not match exactly. This is probably caused by manufacturing tolerances. This causes inhomogeneity in the air supply and the jet directions of the pure water jets and of the pure air jets deviate somewhat from each other.

Compared to the results of the centers of the wetting areas of the straight water injection, larger fluctuations occur with the spray injection. The measurement results of the standard deviations are summarized in Table 2. Here, the largest shot to shot variation occurs for the nozzle in the first column and third row. The largest standard deviation determined is $2.23 \mathrm{~mm}$ (in the vertical direction), which is six times larger than the larges standard deviation for straight water injection. This could be expected, because compared to a pure water jet there are larger fluctuations in a spray jet that occur with an externally mixing nozzle. Nevertheless, the standard deviations are still very small compared to the area sizes and show a good reproducibility. This, in turn, makes it possible to detect even very small changes in the aircarrying system. 
Table 2 - Standard deviations of the positions of the centers of the wetted surfaces for spray injection.

\begin{tabular}{cccll}
\hline column & row & nozzle holes & $\sigma_{\text {hor }}[\mathrm{mm}]$ & $\sigma_{\text {vert }}[\mathrm{mm}]$ \\
\hline \multirow{2}{*}{1} & 1 & large & 0.96 & 2.09 \\
& 2 & small & 0.84 & 1.38 \\
& 3 & large & 1.17 & $\mathbf{2 . 2 3}$ \\
\hline \multirow{2}{*}{2} & 1 & large & 1.00 & 1.14 \\
& 2 & large & 0.60 & 0.99 \\
& 3 & small & 1.39 & 0.93 \\
\hline \multirow{2}{*}{3} & 1 & small & 0.91 & 1.12 \\
& 2 & large & 0.37 & 1.29 \\
& 3 & large & 1.09 & 1.56 \\
\hline
\end{tabular}

More detailed conclusions about the condition of the nozzles can be drawn by combining the measurements in both operating modes - pure water injection and spray injection. Independent changes of the center positions in both operating modes can have different causes and are therefore difficult to interpret. However, a pure change of the alignment of the nozzles leads to an identical change of the center point position in both operating modes. Thus, a pure misalignment can be inferred and corrected. If, in contrast, only the center position changes during spray injection, there must be a problem with the air conveying system and this can be separately be fixed.

\section{Monitoring of nozzle mass flow}

A common problem with nozzles is that the channels gradually become clogged, for example due to release agent deposits. In order to detect when a nozzle reaches a critical state, it would be very advantageous if the flow could be controlled. In preliminary investigations, the mass flow through the nozzles was determined by collecting and weighing the injected water. Here a dependence of the mass flow on the nozzle size and the injection pressure could be determined. The resulting question is now, whether a changing mass flow can also be read in the wetting patterns. Again, here it is not the measurement of the absolute value of the mass flow which is crucial. For a spraying-tool monitoring it is the detection of changes of the mass flow which is of great interest. For this purpose, the size of the wetted areas is used as a characteristic parameter, similar to the mass evaluation in [7].

In the tests, it was found that the size of the wetting areas differs from nozzle to nozzle, while with increasing water pressure the wetting areas are increasing. However, it is known that the large nozzles have a larger mass flow than the small ones. Thus, it must be determined whether the large nozzles have a larger wetting area on average than the small ones. If this is the case, this can be attributed to the larger mass flow. The wetting area is a dynamic parameter and increases with time. To illustrate this, Figure 10 shows the size of the wetting areas for pure water injection versus time. The mean values are derived from the areas of the applied three small nozzles and the six large nozzles bases on 10 repeated injections. After $20 \mathrm{~ms}$ a constant growth can be observed. The fluctuations before are due to the evaluation algorithm, which requires a certain area size for the detection, and due to the successive arrival of the jets. 


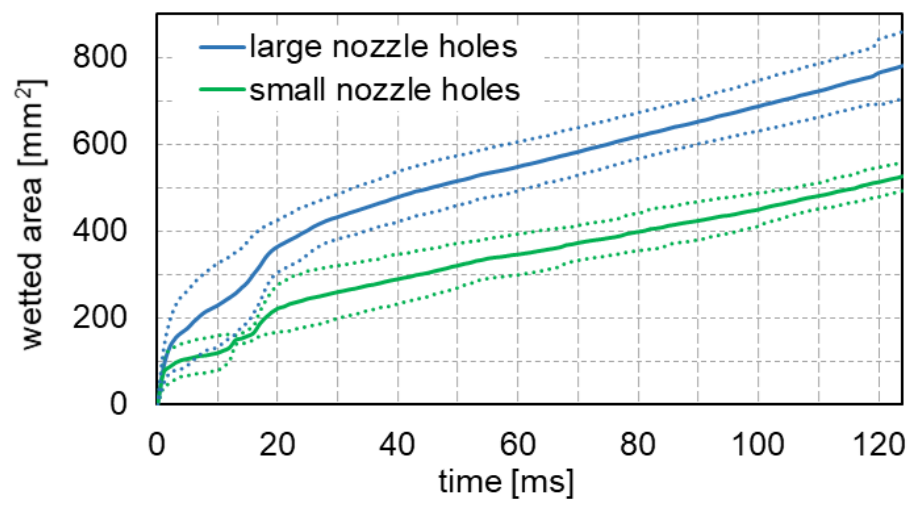

Figure 2. Sizes and standard deviations of the wetted areas over time as average of the areas of 3 small nozzles small and 6 large nozzles applying straight water injection.

The curve shows that the sizes of the wetting areas differ significantly, with the relative standard deviations reaching values of up to $17 \%$. This makes it difficult to clearly assign nozzles on the basis of the wetting pattern at the start of injection. In contrast, individual nozzles can be assigned very reliably to the group of large or small nozzles in the later course from $60 \mathrm{~ms}$. In the case of the wetting area, therefore, a specific evaluation time must be defined. Based on the results, it can be assumed that significant changes in the mass flow can also be detected on the basis of the wetting area.

In addition to the comparative study, the scatter of individual nozzles was also evaluated. At the time of $60 \mathrm{~ms}$, the relative standard deviations of the wetting sizes of individual nozzles show values of approximately $10 \%$. Thus, a reliable detection of the change in mass flow can only be made when the wetting area changes by more than $20 \%$. This statement is correct if only a single test shot is made.

If it turns out in practical operation that smaller mass flow changes are relevant and must be detected, it is necessary to make several test recordings (depending on the required accuracy) and to average the results. This makes it possible to reliably determine even small changes in the range of the standard deviation.

\section{Conclusions}

The regular inspection of the functionality of multi-nozzle spraying tools is important in many industrial applications. Since harsh conditions often prevail in real production operations, the measurement technology used should not only be cost-effective but also robust. It is not always necessary to determine detailed quantitative parameters such as droplet sizes. Instead, it is often essential to determine if the spray has not changed and if the spraying tool behaves as it did when it was first put into operation. To this end, we have developed a simple measurement technique and investigated the performance of this technique in more detail. The technique is based on recording the wetting pattern that occurs during spray-wall interaction on a frosted pane. Such a wetting pattern can now be compared with a master wetting pattern. Differences between the patterns result from unwanted changes in the spraying tool. For our investigations we used a spray tool with nine nozzles.

As a first parameter, we examined the positions of the centers of the wetting areas of each nozzle. For this purpose, we used the spraying tool in different operation modes. Under our boundary conditions and for straight water injection, the maximum standard deviation of the calculated center positions of the wetting surfaces was only $0.35 \mathrm{~mm}$. This means that even the smallest changes in the wetting positions can be precisely detected. If deviations in the 
position of a wetting surface occur, it is possible to assign the associated nozzle and to identify the nozzle alignment or contamination of the water-bearing channels as the cause. Furthermore, we examined the sizes of the wetting areas and found a correlation with the mass flow.

In addition, it is possible to test the air-bearing channels separately, by comparing the distance of the wetting area positions of pure water injection and spray jet injection. If there is a change in this distance between the midpoint positions, a partial malfunction of the air-bearing channels of the belonging nozzle is likely.

Independent of quantitative statements, it is also possible to detect optical, qualitative changes in the wetting pattern. For an automated evaluation, this requires a powerful algorithm. Therefore, further investigations will focus on the development of automated image evaluation. The wetting pattern measurement technique described can be extended with the help of infrared thermography. In addition to position and wetting area information, this also provides locally resolved information on the intensity of the interaction between the spray jet and the surface. Although this method is somewhat more cost-intensive, we expect a simpler evaluation and a larger number of measurement parameters as well as a greater robustness of the measurements against disturbances. The analysis of the IR method is the subject of current investigations.

\section{References}

[1] Dannigkeit, F., Steinke, L., Ripperger, S., 2012, Inline-Messung des mittleren Tropfendurchmessers und der Tropfenkonzentration von Prozesssprays. In: Chemie Ingenieur Technik 84 (3), S. 357-364. DOI: 10.1002/cite.201100201.

[2] Ashgriz, N. (Hg.), 2011, Handbook of Atomization and Sprays. Boston, MA: Springer US.

[3] Mantel, R., 2017, Über die Interaktion fast paralleler sowie kollidierender Kraftstoffsprays. Dissertation. Universitäts- und Landesbibliothek Darmstadt, Darmstadt.

[4] Aísa, L., Garcia, J. A., Cerecedo, L. M.; García Palacín, I., Calvo, E., 2002, Particle concentration and local mass flux measurements in two-phase flows with PDA. Application to a study on the dispersion of spherical particles in a turbulent air jet. In: International Journal of Multiphase Flow 28 (2), S. 301-324. DOI: 10.1016/S0301-9322(01)00071-4.

[5] Shedd, T. A., Newell, T. A., 1998, Automated optical liquid film thickness measurement method. In: Review of Scientific Instruments 69 (12), S. 4205-4213. DOI: 10.1063/1.1149232.

[6] Schulz, F., Reincke, F., Mrochen, M., Beyrau, F., 2021, A measuring system for monitoring multi-nozzle spraying tools. In: Meas. Sci. Technol. DOI: 10.1088/1361-6501/abe082.

[7] Schulz, F, Schmidt, J., Kufferath, A., Samenfink, W., 2014, Gasoline Wall Films and Spray/Wall Interaction Analyzed by Infrared Thermography. In: SAE Int. J. Engines 7 (3), S. 1165-1177. DOI: 10.4271/2014-01-1446. 